\title{
Identification of the Adenine Binding Site in the Ricin Toxin A-Chain by Fluorescence, CD, and Electron Spin Resonance Spectroscopy
}

\section{T. S. RAMALINGAM, ' PUSPENDU K. DAS, ${ }^{1}$ and SUNIL K. PODDER ${ }^{2}$}

Departments of ${ }^{1}$ Inorganic and Physical Chemistry and ${ }^{2}$ Biochemistry, Indian Institute of Science, Bangalore 560 012, India

\begin{abstract}
SYNOPSIS
$\mathrm{CD}$, electron spin resonance, and fluorescence spectroscopy have been utilized to study the adenine binding site of ricin and its toxic A-subunit. At acidic (4.5) and physiological (7.3) $\mathrm{pH}$, adenine or a spin-labeled analogue of adenine, $\mathrm{N}^{6}-(2,2,6,6$-tetramethyl-1-oxypiperidin4-yl) adenine, alters the near uv CD spectra of the ricin A-chain as well as intact ricin, whereas the far uv CD spectra of all proteins remain unchanged. Electron spin resonance data show that the adenine spin-labeled analogue interacts strongly with the A-chain both at $\mathrm{pH} 4.5$ and 7.3, but no or very weak binding is observed for the intact ricin or the isolated $\mathrm{B}$-chain. The adenine spin label gets highly immobilized $\left(2 \mathrm{~A}_{\mathrm{II}}=65.5 \mathrm{G}\right)$ by the A-chain. The apparent dissociation constant $K_{\mathrm{d}}$ for the toxic A-chain ligand complex is 1.55 $\times 10^{-4} \mathrm{M}$ and $5.6 \times 10^{-5} \mathrm{M}$ at $\mathrm{pH} 7.3$ and 4.5 , respectively. Fluorescence intensity of ricin A- chain bound 1,8-anilinonaphthalenesulfonic acid (ANS) decreases by $\sim 55 \%$ at $\mathbf{p H ~} 4.5$ with the addition of the spin-labeled analogue of adenine, implying that both the ANS and adenine spin label (ADSL) bind to the hydrophobic domain of the A-chain. Fluorescence of the only intrinsic tryptophan probe of the A-chain is also efficiently quenched by ADSL, indicating that the tryptophan residue and the hydrophobic adenine binding site are closely located. All spectroscopic measurements indicate that adenine or its spin-labeled analogue has a single binding site adjacent to the TRP211 residue in the A-chain. Expansion of the A-chain globule and subsequent exposure of the hydrophobic binding site seem to be responsible for the increased binding of adenine at pH 4.5. () 1993 John Wiley \& Sons, Inc.
\end{abstract}

\section{INTRODUCTION}

Ricin, a cytotoxic lectin isolated from the Ricinius communis plant, is a glycoprotein made of two nonidentical subunits, an A-chain $\left(30,000 M_{\mathrm{r}}\right)$ and a Bchain $\left(32,000 M_{\mathrm{r}}\right)$ linked by a disulfide bond. The toxic A-chain has been shown to function as N-glycosidase that inactivates $28-\mathrm{S}$ rRNA ribosomal subunits of rat liver in vivo ${ }^{1,2}$ by hydrolyzing a specific adenine from $A_{4324}$ nucleoside residue. The B-chain binds to galactose as well as other oligosaccharides having a terminal galactose ${ }^{3,4}$ Following binding to cell surface carbohydrates, ricin is internalized and the disulfide bond between the A- and the B-chains

Biopolymers, Vol. 33, 1687-1694 (1993)

(C) 1993 John Wiley \& Sons, Inc.

CCC 0006-3525/93/111687-08 is ruptured. It is believed that endosomes at $\mathrm{pH}$ $<5.0$ are probable sites for transmembrane transfer of the A-chain into the cytoplasm. ${ }^{5}$ The free A-chain then exerts its cytotoxic effect by inactivating protein synthesis in eucaryotic systems. Although many hypothesis have been presented, the sequence of events following binding of ricin to the cell surface through the B-chain and ultimate cell death is not known. Recently Wool and co-workers ${ }^{6}$ have shown that the A-chain can depurinate a synthetic GAGA sequence of $16 \mathrm{~S}$ rRNA where the first $\mathrm{A}$ is the target. Monzingo et al. ${ }^{7}$ has shown that adenine or adeninelike pyrazolopyrimidine moiety may be recognized by ricin. Thus binding and recognition of adenine to the A-subunit needs to be addressed. Since this lectin exerts its cytotoxicity presumably by passing through the endosomal site where the $\mathrm{pH}$ of the environment is low, any effect of $\mathrm{pH}$ on its ability to 
bind adenine is, therefore, of immense interest. This protein also has potential applications in cancer therapy as a component of immunotoxins ${ }^{8}$ and is currently an intensely studied system.

It is well established that the B-chain contains the carbohydrate binding domains of ricin. ${ }^{9}$ In addition, ricin has binding domains for hydrophobic ligands, e.g., 1,8-anilinonaphthalenesulfonic acid (ANS), ${ }^{10,11}$ etc. Roberts et al. ${ }^{12}$ have reported that lima bean lectin (LBL) has a single high affinity adenine binding site in addition to binding sites for ANS. Later Maliarik et al. ${ }^{13}$ have established a topographical relation between the adenine binding site and ANS binding domains of lima beans ( Phaseolus lunatus), Dolichos biflorus, and Phaseolus vulgaris lectins. In fact, binding of nonphysiological hydrophobic molecules is common to many proteins. A hydrophobic site of concavalin A (Con A) has been implicated in phorbol ester stimulation of Con A. ${ }^{14}$ For this reason we have decided to investigate the hydrophobic binding properties of ricin and its A- and B-chains using hydrophobic fluorescence probes that have been shown to bind to Con $\mathrm{A}$.

Electron spin resonance (ESR) is a highly sensitive technique for getting information regarding protein-ligand interactions by targeting specific spin-labeled moieties to the interior of a protein. ${ }^{15}$ We have used ESR spectroscopy of a spin-labeled analogue of adenine as a probe to sense the adenine binding pockets in ricin and its A- and B-chains. The ricin A-chain has a single tryptophan residue at position 211 situated at the active site. Ready et al., ${ }^{16}$ from site-directed mutagenesis, have suggested that the interaction between $\operatorname{Trp} 211$ and adenine is crucial for the tight binding of the A-chain to the ribosomes. This encouraged us to use the unique Trp211 as a nonperturbing probe for studying the adenine binding site. CD spectroscopy has been employed to monitor the changes in the secondary and tertiary structures of the proteins induced by the adenine binding.

\section{MATERIALS AND METHODS}

\section{Chemicals}

Adenine, benzyl adenine, 4-amino 2,2,6,6-tetramethylpiperidine-1-oxyl (TEMPO), ANS, and dithiothreitol (DTT) were all obtained from Sigma Chemical Company (USA). The 6-chloropurine was purchased from Fluka (Switzerland). All column materials employed for protein purification were procured from Pharmacia (Sweden).

\section{Protein Purification}

Ricin was isolated from locally available castor bean seeds using the method employed by Lappi et al. ${ }^{17}$ The purity of the protein was checked by sodium dodecyl sulfate-polyacrylamide gel electrophoresis (SDS-PAGE). The disulfide bond connecting the A- and B-chains was cleaved by DTT. The mixture was passed through a lactamyl-sepharose $4 \mathrm{~B}^{18} \mathrm{col}$ umn and the A-chain was eluted with $0.5 M$ Tris $\mathrm{HCl}$ buffer containing $0.5 \mathrm{M} \mathrm{MgCl}_{2}$ salt. ${ }^{19}$ The column-bound $\mathrm{B}$-chain was eluted with the same buffer containing lactose. The purity of the A- and Bchains thus obtained were checked by SDS-PAGE. The gel pattern did not show any evidence for the presence of dimers of either chains. We observed that the isolated A- and B-subunits were unstable after 4 days. Therefore, all experiments were carried out within 2-3 days of isolation.

\section{Synthesis of $N^{6}-(2,2,6,6-T e t r a m e t h y l-1-$ Oxylpiperidin-4-yl)Adenine}

The 6-chloropurine was reacted with 4-amino TEMPO under reflux conditions in an oil bath for about $3 \mathrm{~h}$ as described by Maliarik et al. ${ }^{13}$ The reaction mixture was evaporated to dryness and extracted with ether. The ether-insoluble components were separated by passing through a silica gel column using chloroform-methanol $(9: 1)$ as the developing solvent. Red color crystals ( $\mathrm{mp} 221-222^{\circ} \mathrm{C}$ ) were obtained with $\sim 72 \%$ yield. The purity of the compound was determined by thin layer chromatography and mass spectroscopy.

\section{ESR Measurements}

ESR spectra were taken on a Varian E-9 spectrometer in microcapillary tubes at room temperature. All spectra were measured at X-band $(9.05 \mathrm{GHz})$ with $20 \mathrm{~mW}$ microwave power. The $100 \mathrm{G}$ scan range was used in most experiments. High gain spectra were obtained at 5-10 times the receiver gain and a modulation amplitude of $2-4 \mathrm{G}$ in order to resolve the bound components at the wings of the spectrum.

\section{Fluorescence Measurements}

Fluorescence spectra were recorded in a microprocessor-controlled Shimadzu RF-5000 spectrofluorimeter at room temperature. ANS fluorescence was monitored by exciting at $420 \mathrm{~nm}$ with the excitation and emission bandwidths of 2.5 and $5 \mathrm{~nm}$, respectively. ANS bound protein was titrated with small volumes of spin-labeled adenine. A blank titration 
was done with ANS in the absence of lectin. The fluorescence spectrum was corrected for contribution from the blank and normalized with respect to the fluorescence of ANS bound lectin in the absence of adenine spin label (ADSL).

Tryptophan emission was recorded by exciting at $295 \mathrm{~nm}$ with both excitation and emission bandwidths set at $5 \mathrm{~nm}$. The proteins were titrated with its spin-labeled analogue by adding small aliquots of the spin probe, keeping the protein concentration constant throughout the experiment. Fluorescence quantum yield of tryptophan was calculated, assuming a quantum yield value of 0.2 for free tryptophan in aqueous solution. ${ }^{20}$ Protein concentration was calculated by measuring the absorbance at 280 $\mathrm{nm}$. The extinction coefficients $\left(\epsilon_{280}^{1 \%}\right)$ of ricin and its polypeptide chains are taken as 7.65 for the Achain, 14.9 for the B-chain, and $\mathbf{1 1 . 7}$ for the intact ricin. $^{21}$

\section{Measurements}

CD spectra were measured at room temperature in a JASCO-5000 spectropolarimeter equipped with a variable temperature accessory. Near uv CD spectra were recorded with a 5 -mm cuvette. Far uv CD spectra were taken with a $1-\mathrm{mm}$ cell. Low noise near uv CD spectra were obtained by averaging over 8-16 scans. For near uv CD titrations, small aliquots of adenine or ADSL were added to a fixed concentration of the protein. A parallel titration was done by adding the spin-labeled analogue to the buffer in the absence of protein. Corrections were made for the blank $\mathrm{CD}$ absorption. The $\theta_{\mathrm{mr}}$ or the mean residue ellipticity in deg $\mathrm{cm}^{2} \mathrm{dmol}^{-1}$, was calculated from the observed ellipticity $\theta$ (in mdeg) assuming a mean residue molecular weight of 110 for the A-chain, 109 for the B-chain, ${ }^{22}$ and 109.6 for intact ricin. ${ }^{23}$

\section{RESULTS}

\section{ESR Data}

The ESR spectra recorded at $\mathrm{pH} 4.5$ for ADSL in the presence of the ricin A-chain are displayed in Figure 1a-c. In addition to the reduction in the ESR signal intensity without any change in the line shape, an immobilized component appears at the wings of the spectrum at a very high gain. Addition of excess adenine to the ADSL + A-chain mixture abolishes the immobilized component completely accompanied by a partial recovery of the signal intensity. Addition of the A-chain produces a large reduction in the free label ESR signal intensity also at $\mathrm{pH} 7.3$

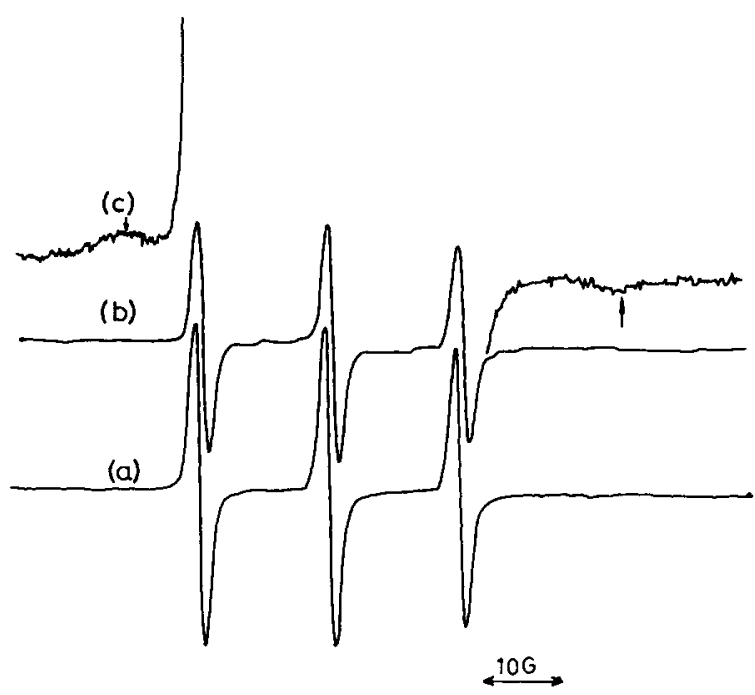

Figure 1. Adenine spin label spectra with ricin A-chain at pH 4.5 (a) free spin label $(65 \mu M)$, (b) after addition of ricin $\mathrm{A}(140 \mu M)$, and (c) at a higher gain. Hyperfine extrema separation is marked by arrows.

(data not shown). But no immobilized component is visible even at the highest gain. From the ESR titrations, ADSL binding to the A-chain is apparent. In contrast, the reduction in the free ADSL signal intensity was marginal after addition of ricin at both pH 7.3 and 4.5 (data not shown), indicating, at best, a weak binding between the spin label and the protein.

Scatchard analysis of the ESR titrations were done for protein binding to the adenine spin label at $\mathrm{pH} 7.3$ and 4.5 (Figure 2a,b) and the values were fit to a straight line by least-squares method. The binding constants for the A-chain with ADSL thus estimated are listed in Table I and are accurate within $10-15 \%$ of the true value of the adenine binding constant. It was not possible to calculate the $K_{\mathrm{d}}$ values for intact ricin since the reduction in ESR signal intensity was not sufficient to quantitate the observed results. From the $x$-axis intercepts ( $n$ $=0.75$ at $\mathrm{pH} 4.5$ and $n=0.72$ at $\mathrm{pH} 7.3$ ) of the Scatchard plots, it is clear that approximately one binding site for adenine exists in the ricin A-chain at either $\mathrm{pH}$. A stronger affinity for adenine at the acidic $\mathrm{pH}$ compared to the physiological $\mathrm{pH}$ is apparent from the binding constant values. Addition of excess ANS to the ADSL + A-chain mixture eliminated the immobilized component followed by a partial recovery of the free label signal intensity.

\section{Fluorescence Measurements}

The fluorescence titration of ricin A-chain bound ANS with adenine spin label shows a decrease in 

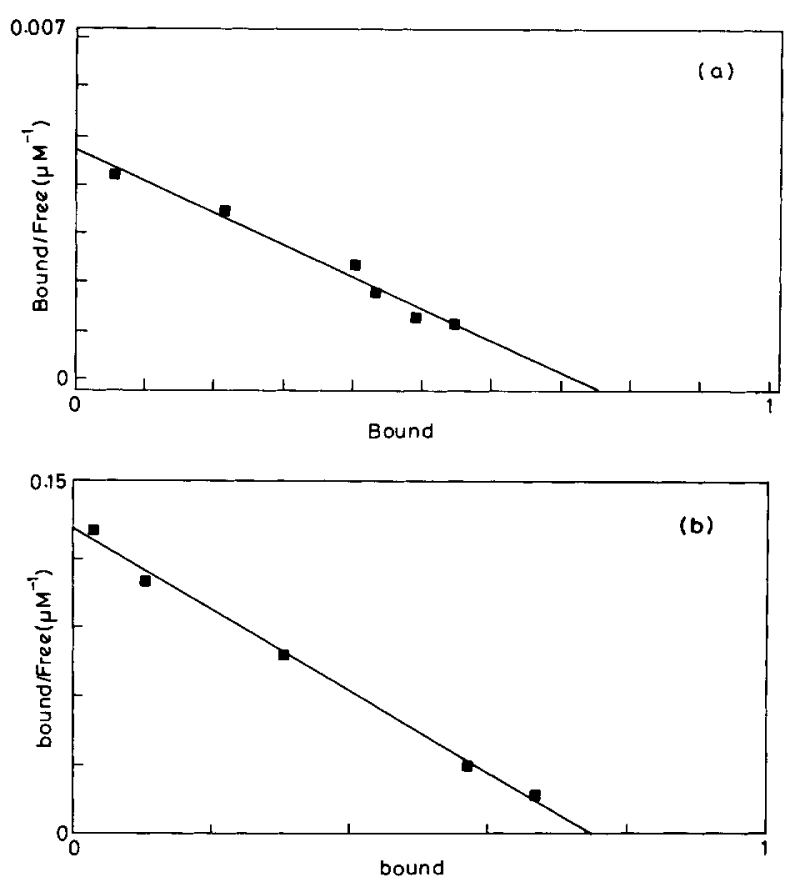

Figure 2. Scatchard plot of adenine spin label binding to ricin $\mathrm{A}$. The straight line through the data points is a least-squares fit. (a) ADSL (12-520 $\mu M$ ) and ricin A (250 $\mu M$ ) at pH 7.3; (b) ADSL $(2.5-400 \mu M)$ and ricin A (270 $\mu M)$ at $\mathrm{pH} 4.5$.

fluorescence intensity at both acidic (4.5) and physiological (7.3) pH (Figure 3). The maximum extent of quenching was $\sim 55 \%$. But the decrease was less dramatic under neutral conditions. The inset of Figure 3 shows the relative fluorescence intensity plot of bound ANS as a function of the spin label concentration. Similar titrations of ANS bound ricin and ricin B-chain with ADSL resulted in a small decrease in fluorescence intensity as a function of spin label concentration. We have recorded the ESR spectrum of ADSL in the presence of ANS tagged ricin A-chain to check whether the fluorescent moiety affects the adenine binding ability of the protein. The signal intensity decreases at acidic as

Table I Binding Parameters of ADSL to the Ricin A-chain and Intact Ricin

\begin{tabular}{lcc}
\hline \multicolumn{1}{c}{ Protein } & $\begin{array}{c}K_{\mathrm{d}}\left(\times 10^{5}\right) \\
(M)\end{array}$ & $\begin{array}{c}2 \mathrm{~A}_{\mathrm{II}} \\
(\mathrm{G})\end{array}$ \\
\hline Ricin & $\mathrm{ND}^{\mathrm{a}}$ & $\mathrm{ND}$ \\
Ricin A-chain (pH 7.3) & $15.5(34.5)^{\mathrm{b}}$ & ND \\
Ricin A-chain (pH 4.5) & $5.6(3.6)^{\mathrm{b}}$ & 65.5 \\
\hline
\end{tabular}

ND: not determined.

${ }^{b}$ Obtained from tryptophan fluorescence quenching by ADSL.

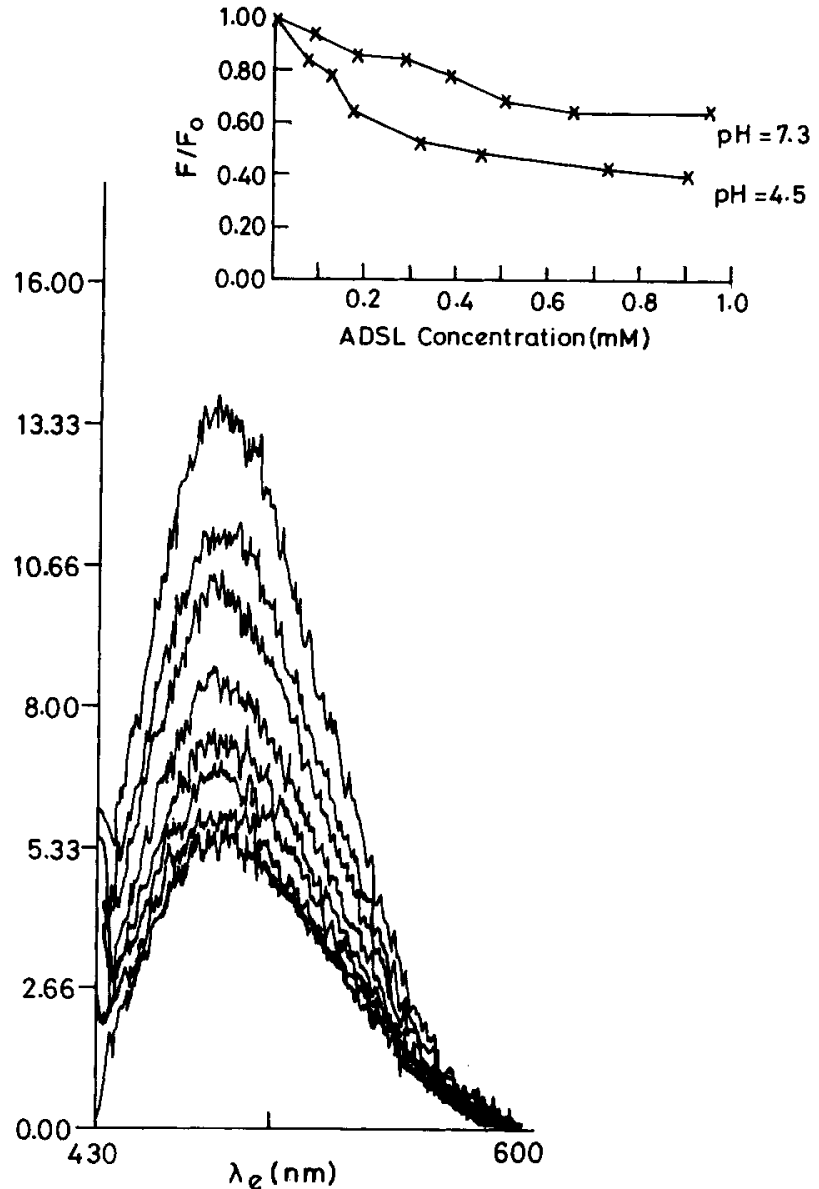

Figure 3. Decrease in protein bound ANS fluorescence by spin-labeled adenine. The spectra were recorded at $\mathbf{p H}$ 4.5 at room temperature ( $\mathrm{ANS} ; 32 \mu M$; ricin A; $51 \mu M$ ) in acetate buffer. Inset is a titration of ANS bound ricin A against adenine spin label at two different $\mathrm{pH}$.

well as neutral $\mathrm{pH}$, implying the retention of adenine binding ability of the lectin even in the presence of the fluorescent marker.

Ricin and ricin B-chain display a fluorescence maximum at $331 \mathrm{~nm}$ whereas the A-chain shows a maximum at $329 \mathrm{~nm}$ when excited at $295 \mathrm{~nm}$. The quantum yield calculated for tryptophan in the Achain at neutral and acidic $\mathrm{pH}$ are 0.27 and 0.25 , respectively, which agrees very well with those reported by Bushueva et al. ${ }^{24}$ Figure 4 shows the decrease in relative fluorescence intensity of the only tryptophan residue of the toxic A-chain with increase in the ADSL concentration. The plot shows that quenching is much more effective at the acidic $\mathrm{pH}$. Scatchard analysis of the fluorescence quenching data (not shown) were done and $K_{\mathrm{d}}=3.6$ $\times 10^{-5} M$ at pH $4.5(n=0.96)$ and $K_{\mathrm{d}}=3.45$ $\times 10^{-4}(n=0.71)$ at $\mathrm{pH} 7.3$ are obtained. These 


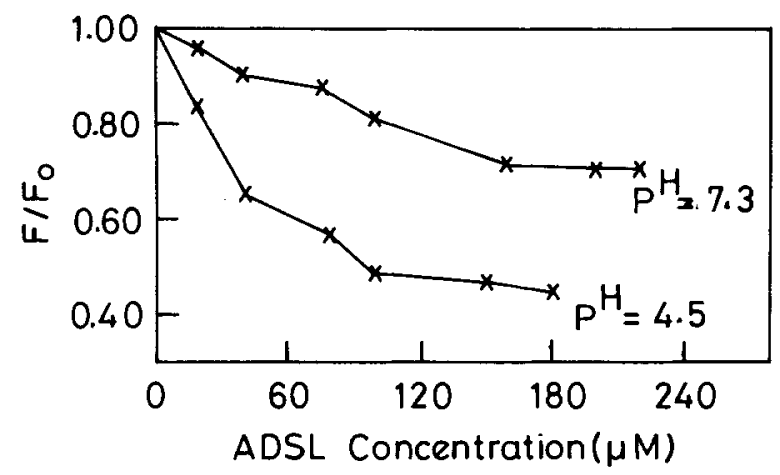

Figure 4. Decrease in normalized tryptophan fluorescence by spin labeled adenine: $150 \mu M$ ricin $A$ in $600 \mu \mathrm{L}$ phosphate buffer (pH 7.3) and $160 \mu M$ ricin A in $600 \mu \mathrm{L}$ acetate buffer ( $\mathrm{pH} 4.5)$.

values compare well with those obtained from the ESR results (Table I). In order to establish that the observed fluorescence quenching is not due to collisional interaction between the free radical and the tryptophan chromophore, we carried out a parallel titration with tempamine in the presence of the ricin A-chain. No quenching of fluorescence is noticed, which means that the tryptophan is, indeed, involved in the adenine binding site and interacts strongly with ADSL when excited.

\section{Measurements}

Figure 5 shows the near uv CD spectrum of intact ricin with two broad bands showing contributions from the Trp and Tyr residues..$^{23}$ Addition of adenine produces a considerable increase in the ellipticity. With increasing concentrations of adenine, the tryptophan and tyrosine bands merge, resulting in a common broad band in that region. Addition of adenine spin label produces an increase in the negative band without altering any other feature of the spectrum. However, when benzyl adenine is added to the mixture, the structural pattern returns, but with a shift in the tyrosine absorption position, leaving the tryptophan peak position unchanged. Addition of excess benzyl adenine produces a large increase in the -ve CD band of tyrosine. These changes are also observed at $\mathrm{pH} 4.5$.

In contrast, the A-chain at $\mathrm{pH} 4.5$ gives a + ve $\mathrm{CD}$ absorption band in the near uv centered around $280 \mathrm{~nm}$ (Figure 6). Addition of adenine produces another +ve CD band at $265 \mathrm{~nm}$. This band disappears by addition of excess benzyl adenine. But a new CD band at $263 \mathrm{~nm}$ with a -ve ellipticity appears. ADSL addition at this stage results in an increase of the tve CD band. Similar experiments carried out at neutral $\mathrm{pH}$ show less dramatic changes.

The ricin A-chain displays (Figure 7) a similar far uv CD spectrum to that of intact ricin, except it has a slightly more pronounced shoulder at $220 \mathrm{~nm}$ than ricin at either $\mathrm{pH}$. Addition of adenine and its spin-labeled analogue marginally alters the spectrum. Ricin and its B-chain also behave in a similar manner. These results imply that the binding of adenine does not induce any significant alterations in their secondary structures.

\section{DISCUSSION}

In this paper we have examined adenine binding sites in ricin and its constituent subunits using

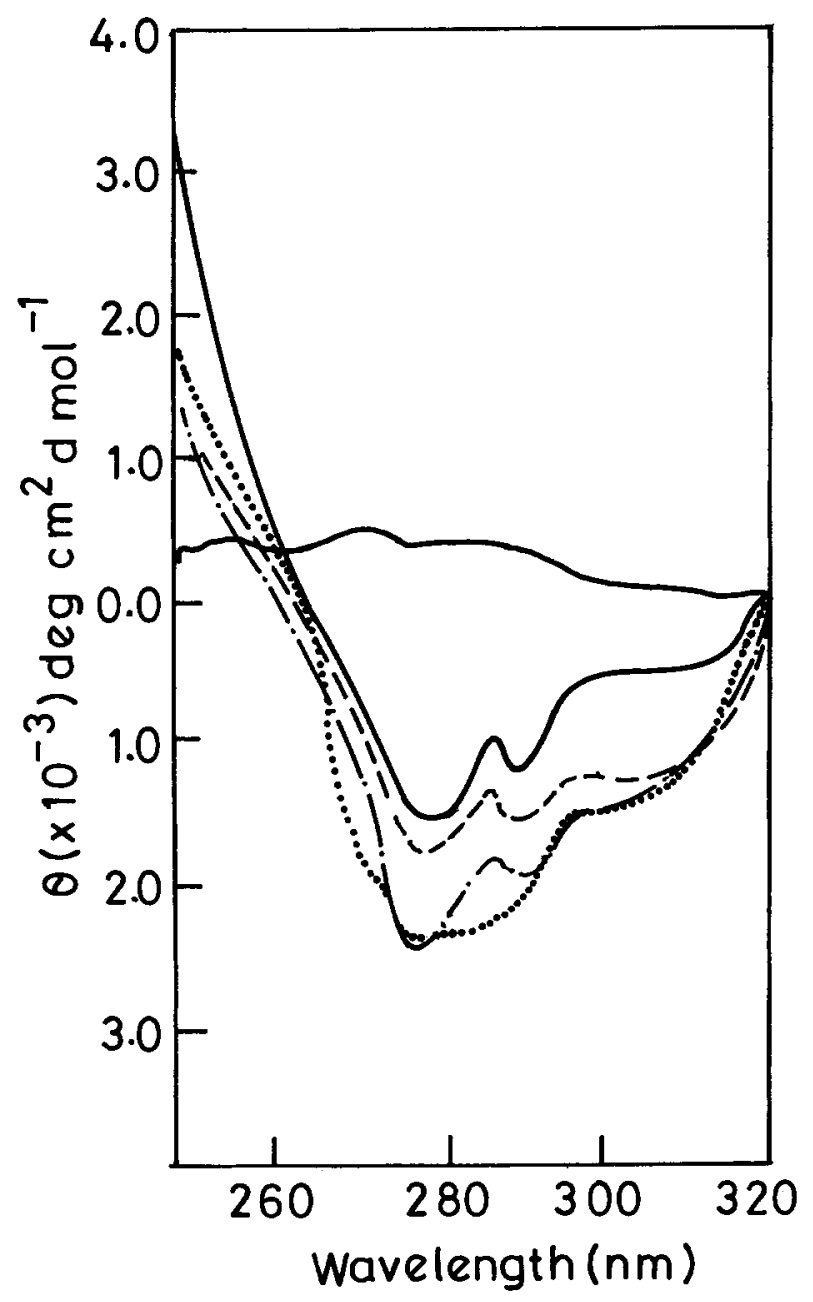

Figure 5. Near uv CD spectra of intact ricin at pH 7.3: $10 \mu M$ ricin (solid line), with $140 \mu M$ adenine (dotted line), plus $320 \mu M$ benzyl adenine (broken line), and after addition of an additional $340 \mu M$ benzyl adenine (dotdash line). 
spectroscopic techniques. The affinity constants of the adenine binding to the A-chain at two different pHs have been determined using ESR titrations of an equivalent adenine spin label with lectin. The binding affinity is large $\left(K_{\mathrm{a}}=1.79 \times 10^{4} \mathrm{M}^{-1}\right.$ at $\mathrm{pH}$ 4.5 ) and is at least two orders of magnitude higher for the A-chain than the carbohydrate binding Bchain or the intact ricin molecule. It is also found that the A-chain has a single high affinity binding site for adenine at both $\mathrm{pH} 4.5$ and 7.3. The immobilized component between ADSL and the Achain at low $\mathrm{pH}$ indicates the presence of an extended hydrophobic adenine binding pocket that helps accommodate the adenine head and its spinlabeled tail in such a fashion as to prevent the free rotational motion of the nitroxypiperidinyl moiety. Addition of free adenine or ANS, however, removes this component. ESR as well as fluorescence quenching experiments show that both ANS and ADSL bind to the hydrophobic surfaces of the lectins and ADSL either replaces ANS from its site (competitive binding) or has negative cooperativity in binding to adjacent sites in the ricin A-chain.

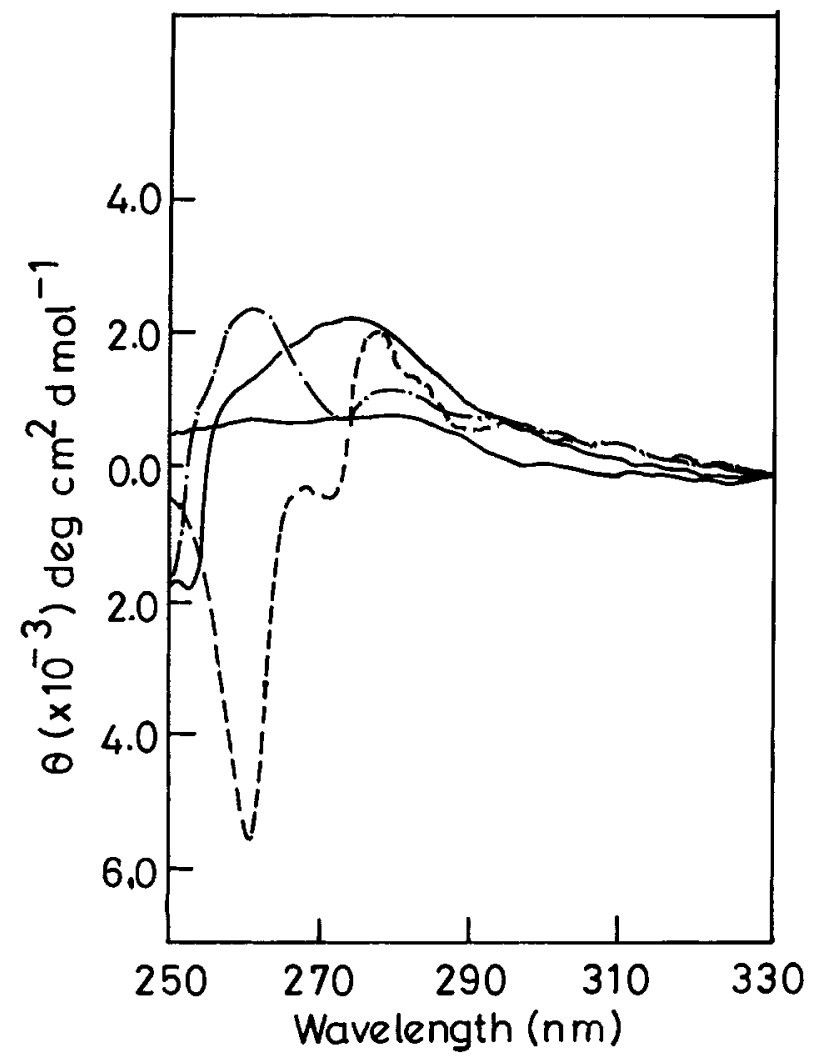

Figure 6. Near uv CD spectra of ricin A at pH 4.5: 18.3 $\mu M$ ricin A (solid line), plus $180 \mu M$ adenine free base (dot-dash line), and after addition of $220 \mu M$ benzyl adenine to the mixture (broken line).

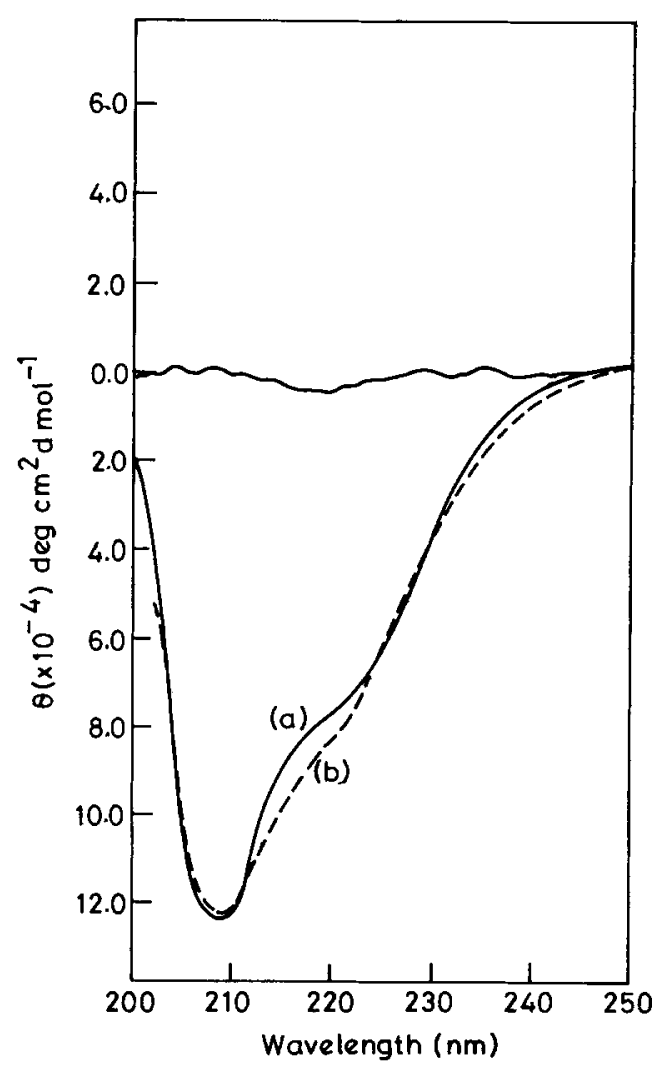

Figure 7. Far uv CD spectra of (a) ricin A (10 $\mu M)$ and (b) plus adenine $(420 \mu M)$ at $\mathrm{pH} 7.3$.

Far uv CD studies show that the adenine binding does not produce any major alterations in the secondary structure of ricin or its contituent polypeptides. However, the near uv CD studies indicate structural reorganization in the proteins involving Tyr and Trp residues. Therefore, at the endosomal compartment at $\mathrm{pH}<5.0$ it is conceivable that the A-chain exposes its hydrophobic domains to the solvent. ${ }^{25-28}$ Consequently, aromatic ligands including adenine are stabilized in these domains by hydrophobic contacts.

The quenching of $55 \%$ of the total fluorescence intensity of the sole tryptophan of ricin A with ADSL at $\mathrm{pH} 4.5$ indicates that the adenine binding site is situated nearby. The increase in $\theta_{278}$ with addition of excess benzyl adenine reveals that it binds to a site close to the adenine binding site. The observed differences in the spectral pattern, however, is possibly due to the more apolar nature of benzyl adenine than ADSL. Katzin et $a{ }^{28}$ have predicted that the active site of the A-chain contains aromatic groups that tend to pack edgewise. Hence cooperative interaction between adenine and these moities is likely. In the crystalline state the active site is 
identified as a cleft containing several invariant amino acids. It has two tyrosine (Tyr80 and Tyr123) and one tryptophan (Trp211) residues and is hydrophobic in nature. Morris et al. ${ }^{29}$ has supported this recently by reporting that the activity of the toxic A-subunit is reduced upon deletion of one of these residues from the chain. The perturbation of these residues after adenine binding is clear from $\mathrm{CD}$ as well as fluorescence quenching data. Therefore, exposure of the active site with conformational alterations in solution must be responsible for the difference in adenine binding affinity of ricin and its A-subunit. But why is adenine binding to the Achain enhanced by twofold at the acidic $\mathrm{pH}$ ? This may be due to two reasons: (1) expansion of the Achain globule at $\mathrm{pH} 4.5$, which exposes the hydrophobic adenine binding site to the incoming ligand; and (2) protonation of certain amino acids under acidic conditions at the binding site region, which in turn makes the local environment more hydrophobic. The first possibility is conceivable since the secondary structure remains largely unaltered after addition of adenine. The cleft identified as the active site region in the $\mathrm{A}$-chain becomes exposed to the aqueous medium as a result of the change in the tertiary structure of the protein. The second possibility exists since the active site in ricin $A$ has the invariant residues Tyr80, Tyr123, Glu177, Arg180, and Trp211, and the glutamic acid residue may get protonated in an acidic medium. Alternatively, another conserved active site with residues Asn78, Arg134, Gln173, Glu208, and Asn209 may play a more specific role at an acidic medium. Glu208 may be protonated and the wall of the active site cleft becomes more hydrophobic to facilitate adenine binding.

\section{CONCLUDING REMARKS}

We have established in this paper from comparative studies among the plant toxin ricin and its $\mathrm{A}$ - and B-chains the following:

1. The A-chain has one adenine binding site in the active region, which is in agreement with the earlier prediction of Katzin et al. ${ }^{28}$ from the Fourier computed difference electron density map of diffused adenine in the ricin crystal. However, at the resolution at which their experiment was done it appeared that the binding site could accommodate two adenine molecules. The A chain binds strongly to adenine at $\mathrm{pH} 4.5\left(K_{\mathrm{d}}=5.6 \times 10^{-5} \mathrm{M}\right)$ but weakly at $\mathrm{pH} 7.3\left(K_{\mathrm{d}}=1.55 \times 10^{-4} M\right)$. Intact ricin at best binds adenine very feebly $\left(K_{\mathrm{d}}\right.$ $<10^{-2} \mathrm{M}$ ).

2. The rupture of the disulfide bond in ricin exposes the hydrophobic binding domains to incoming aromatic ligands, and the binding of ANS, adenine, and benzyl adenine is competitive or has a negative cooperativity. The active site Trp211 residue is in the proximity of this hydrophobic adenine binding site.

Finally, adenine binding appears to be more complicated than carbohydrate binding in ricin. The invariant residues at the active site of ricin $A$ are common to all toxin sequences known and therefore should be targeted to site-specific mutagenesis efforts to pinpoint the mechanism of catalytic action. Our studies indicate that for an effective binding to ribosomes the hydrophobic stabilization of the adenine substrate at the active site is one of the important factors for its catalytic activity.

One of us (SKP) would like to thank The Council of Scientific and Industrial Research, Government of India, for partly funding this research. We also thank Prof. K. R. K. Easwaran for making the CD spectrometer available to us, and Profs. P. Balaram, V. Raghavan, and S. Bhattacharyya for many helpful discussions.

\section{REFERENCES}

1. Endo, Y., Mitsui, K., Motizuki, M. \& Tsurugi, K. (1987) J. Biol. Chem. 262, 5908-5912.

2. Endo, Y. \& Tsurugi, K. (1987) J. Biol. Chem. 262, 8128-8130.

3. Olsnes, S. \& Pihl, A. (1973) Biochemistry 12, 31213126.

4. Olsnes, S. \& Pihl, A. (1982) in Molecular Action of Toxin And Viruses, Cohen, P. \& van Heyningen, S., Eds., Elsevier, Amsterdam, pp. 51-105.

5. Blum, J. S., Fiani, M. L. \& Stahl, P. D. (1991) J. Biol. Chem. 266, 22091-22095.

6. Endo Y., Chan, Y. L., Lin, A., Tsurugi, K. \& Wool, I. G. (1988) J. Biol. Chem. 263, 7917-7920.

7. Monzingo, A. F. \& Robertus, J. D. (1992) J. Mol. Biol. 227, 1136-1145.

8. Blattler, W. A., Lambert, J. M. \& Goldmacher, V. S. (1989) Cancer Cells 1, 50-55.

9. Funatsu, G., Kimura, M. \& Funatsu, M. (1979) Agricul. Biol. Chem. 43, 2221-2224.

10. Halling, K. C., Halling, A. C., Murray, E. E., Ladin, B. F. \& Houston, L. L. (1980) Biochem. Biophys. Res. Commun. 92, 319-326.

11. Roberts, D. D. \& Goldstein, I. J. (1982) J. Biol. Chem. 257, 11274-11277. 
12. Roberts, D. D. \& Goldstein, I. J. (1983) J. Biol. Chem. 258, 13820-13824.

13. Maliarik, M. J., Plessas, N. R., Goldstein, I. J., Musci, G. \& Berliner, L. J. (1989) Biochemistry 28, 912-917.

14. Edelman, G. M. \& Wang, J. L. (1978) J. Biol. Chem. 253, 3016-3022.

15. Berliner, L. J. (1982) in Spectroscopy and Biochemistry Bell, J. E., Ed., Vol. 2, CRC Press, West Palm.Beach, FL.

17. Lappi, D. A., Kapmeyer, W., Beglau, J. M. \& Kaplan, N. O. (1978) Proc. Natl. Acad. Sci. USA 74, 10961100 .

18. Matsumoto, I., Kitagaki, H., Akai, Y., Ito, Y. \& Seno, N. (1981) Anal. Biochem. 116, 103-110.

19. Emmanuel, F., Turpin, E., Alfsen, A. \& Frenoy, J. P. (1988) Anal. Biochem. 173, 134-141.

20. Teale, F. W. J. \& Weber, G. (1957) Biochem. J. 65, 472-482.

21. Olsnes, S., Slatvedt, E. \& Pihl, A. (1974) J. Biol. Chem. 249, 803-810.
22. Wawrzynczak, E. J., Drake, A. F. \& Thorpe, P. E. (1988) Biophys. Chem. 31, 301-305.

23. Frenoy, J. P. (1986) Biochem. J. 240, 221-226.

24. Bushueva, T. L. \& Tonevitsky, A. G. (1987) FEBS. Lett. 215, 155-159.

25. Lewis, M. S. \& Youle, R. J. (1986) J. Biol. Chem. 261, 11571-11577.

26. Montfort, W., Villafranca, J. E., Monzingo, A. T., Ernst, S. R., Katzin, B., Rutenber, E., Xuong, N. H., Hamlin, R. \& Robertus, J. D. (1987) J. Biol. Chem. 262, 5398-5403.

27. Rutenber, E. \& Robertus, J. D. (1989) in Trans. Amer. Cryst. Assoc. 25, 77-85.

28. Katzin, B. J., Collins, E. J. \& Robertus, J. D. (1991) Proteins Struct. Funct. Genet. 10, 251-259.

29. Morris, K. N. \& Wool, I. G. (1992) Proc. Natl. Acad. Sci. USA 89, 4469-4473.

Received January 12, 1993

Accepted April 27, 1993 